Research Article

\title{
Fractal Loading Model of the Joint Interface Considering Strain Hardening of Materials
}

\author{
Yanhui Wang $\mathbb{D}^{1,2}$ Xueliang Zhang $\mathbb{D}^{1},{ }^{1}$ Shuhua Wen, ${ }^{1}$ and Yonghui Chen ${ }^{1}$ \\ ${ }^{1}$ School of Mechanical Engineering, Taiyuan University of Science and Technology, Taiyuan 030024, China \\ ${ }^{2}$ Department of Mechanical and Electrical Engineering, Shanxi Institute of Energy, Jinzhong 030600, China
}

Correspondence should be addressed to Xueliang Zhang; zhang_xue_l@sina.com

Received 2 August 2018; Revised 7 December 2018; Accepted 6 February 2019; Published 3 March 2019

Academic Editor: Fabio Minghini

Copyright ( 12019 Yanhui Wang et al. This is an open access article distributed under the Creative Commons Attribution License, which permits unrestricted use, distribution, and reproduction in any medium, provided the original work is properly cited.

Based on fractal geometry theory, the deformation state of the four stages of the asperity elastic, first elastoplastic, second elastoplastic, and fully plastic deformation and comprehensively considering the hardness of the asperity changes with the amount of deformation in elastoplastic deformation stage due to strain hardening are considered, thereby establishing a single-loading model of the joint interface. By introducing the pushing coefficient and the asperity frequency exponent, each critical frequency exponents of asperity is obtained, and the relationship between the normal contact load and the contact area of the first elastoplastic deformation phase and the second elastoplastic deformation phase of the single asperity in the case of taking into account the change in hardness is inferred, eventually deducing the relationship between the total contact load of the joint interface and the contact area. The analysis results show that in the elastoplastic deformation stage, when the deformation is constant, the asperity load considering the hardness change is smaller than the unconsidered load, and the difference increases with the increase of the deformation amount. The establishment of the model provides a theoretical basis for further research on the elastoplastic contact of joint interfaces.

\section{Introduction}

The joint formed by assembly of mechanical parts is called the joint interface, which plays an important role in the transmission of motion, load, and energy in the normal operation of mechanical system. The joint interface presents a series of different curvature radius and the height asperities at the microscopic scale. The contact between the joint interface is discontinuous and only occurs at higher asperity, lead to the real contact area accounts for only a small part of the nominal contact area, resulting in the situation of large load on a small contact area. As a result, researches on the properties of interfacial contact and stress analysis are very complex [1]. Therefore, to study the deformation behavior and the accurate modeling of joint interface is an important issue for in-depth understanding of the mechanism of friction, wear, lubrication, heat conduction, etc.

Statistical and fractal contact models for solving contact problems on joint interface have been used widely in this field. Statistical contact model was originally put forward by Greenwood and Williamson (GW model) and improved by many subsequent researchers [2-4]. Zhao et al. deduced a new elastoplastic contact model of joint interface, which describing a long transition period from elastic deformation to fully plastic deformation of joint interface. It is shown that the elastoplastic contact of the asperity plays an important role in the microscopic contact behavior of the joint interface [5]. Kogut and Etsion established the contact model between a single asperity and a rigid flat by means of finite element analysis and obtained the relationship between contact area and contact load of a single asperity during loading and unloading [6, 7]. Kadin et al. applied the conclusion of Etsion to the whole joint interface and got a statistical model of single loading and unloading of joint interface. According to his conclusion, plastic deformation and residual stress may occur in the process of loading and unloading. The actual contact area of the asperity during unloading is larger than the actual contact area of the loading 
process $[8,9]$. However, the value of statistical parameters depends largely on the filter or resolution of the roughness measuring instrument, so it is not unique for a joint interface.

The fractal model was first proposed by Majumdar and Bhushan (MB model) in 1990. The model holds that the deformation of microconvex body changes from plastic deformation to elastic deformation with the increase of load, contrary to the traditional contact study [10-12]. Many scholars put forward many kinds of fractal models based on MB fractal model and obtained more accurate contact mechanical properties of joint interface. Wang et al. modified the area distribution density function of asperity in $\mathrm{MB}$ model and obtained the modified model of $\mathrm{MB}$ elastic and plastic contact [13, 14]. Morag and Etsion established the elastoplastic contact fractal model of a single asperity and explained the contradiction between the deformation sequence of asperity from plastic deformation to elastic deformation in MB model and the classical Hertz contact theory [15]. Tian et al. modified the model further, taking into account the change of material hardness with the change of surface depth in elastoplastic stage and established a new single-loading model of joint interface. However, the model only takes into account the transition from elastic to elastoplastic and elastoplastic to fully plastic deformation stage of the asperity. The description of the elastoplastic deformation stage is seldom involved in the model [16]. Yuan et al. proposed an improved model of the fractal elastoplastic contact model of rough surface based on the MB model, so as to deduce a model of the total contact load and the total actual contact area [17]. However, the model does not take into account the strain hardening phenomenon of the joint interface material, that is, the hardness of the material is no longer a constant value, but will change with the increase of the amount of deformation. Hardness is an important index to characterize the mechanical properties of materials such as elasticity, plasticity, strength, and toughness. The change of hardness value is directly related to the accuracy of calculation. According to the strain hardening criterion, the average hardness increases with the increase of deformation. The degree of plastic deformation increases, the degree of work hardening and dislocation strengthening increases, and the hardness of the material increases. Based on the above research results and fractal theory, a new hardness change function is expected to be constructed in this paper, considering that the hardness of the material changes with the deformation amount of the asperity in the elastoplastic deformation stage. In this paper, the critical conditions of elastic, elastoplastic, and plastic deformation of asperity are studied, and the four deformation ranges are revised and a fractal theoretical model describing the single loading of the joint interface is proposed. It is expected that the microscopic and macroscopic contact state of the surface of the interface can be more scientifically and reasonably described in order to provide some theoretical basis for the research of contact, friction, wear, and lubrication on the surface of mechanical parts.

\section{Fractal Model of a Single Asperity}

Majumdar et al. show that the contours of joint interface topography in practical engineering have fractal characteristics; mathematical characteristics are continuity, nondifferentiability, and self-affinity $[10,11]$. The joint interface profile can be described by the Weierstrass-Mandelbrot (WM) function, which is expressed as

$$
Z(x)=G^{(D-1)} \sum_{n=n_{\min }}^{\infty} \frac{\cos \left(2 \pi \gamma^{n} x\right)}{\gamma^{(2-D) n}}, \quad(1<D<2, \gamma>1),
$$

where $x$ is the horizontal coordinate of the profile function of the joint interface, and the corresponding function value is the height of the profile; $D$ is the fractal dimension of the surface profile (for a physically continuous surface, $1<D<2$ ); $G$ is the length scale parameter of the surface, which reflects the amplitude of $Z(x)$, and is the measurement constant; $n_{\min }$ is the lowest frequency exponent corresponding to the profile; and $\gamma^{n}$ determines the spectrum of surface roughness, which is the frequency density control parameter, $\gamma>1$. The actual surface profile has an unstable randomness [18], and its lowest frequency is related to the sample length, which is given by $\gamma^{n_{\min }}=1 / L$. In order to satisfy the requirements for high spectral density and for phase randomization, $\gamma=1.5$.

2.1. Elastic Deformation of a Single Asperity. On the microscopic scale, the contact between the two joint interfaces is essentially a contact between the asperity and the asperity, which can be simplified as a contact between a series of equivalent asperities on the joint interface and a rigid flat surface. Assuming that the joint interface is isotropic, there is no interaction between the asperity and the asperity during the contact process, and no large deformation will occur. The equation before deformation of the asperity with frequency exponent $n$ is obtained as follows:

$$
z_{\mathrm{n}}(x)=G^{D-1} \frac{\cos \left(\pi \gamma^{n} x\right)}{\gamma^{(2-D) n}}\left(-\frac{1}{2 \gamma^{n}}<x<\frac{1}{2 \gamma^{n}}\right) .
$$

Figure 1 shows an asperity in equivalent joint interface contacts with a rigid flat surface. The height of the asperity is $h_{\mathrm{n}}$, the interference of the asperity is $\omega_{\mathrm{n}}$ during the loading process, and the size of the substrate of the asperity is $l_{n}$. According to equation (2), the curvature radius of an asperity with frequency exponent $n$ at any point $x$ is obtained as follows:

$$
\begin{aligned}
\rho_{\mathrm{n}}(x) & =\left|\frac{\left\{1+\left[d z_{\mathrm{n}}(x) / d x\right]^{2}\right\}^{3 / 2}}{d^{2} z_{\mathrm{n}}(x) / d x^{2}}\right| \\
& =\frac{\left[1+\left(\pi^{2} G^{2(D-1)} / \gamma^{2(1-D) n}\right) \sin ^{2}\left(\pi \gamma^{n} x\right)\right]^{3 / 2}}{\left|-\left(\pi^{2} G^{D-1} / \gamma^{-D n}\right) \cos \left(\pi \gamma^{n} x\right)\right|} .
\end{aligned}
$$

When $x=0$, the curvature radius of asperity is minimum: 


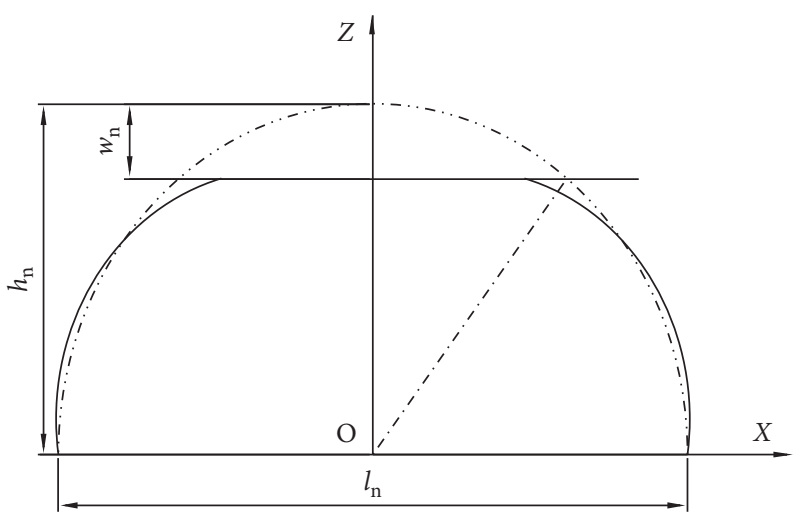

Figure 1: Diagram of single asperity loading.

$$
\rho_{\mathrm{nmin}}(x)=R_{\mathrm{n}}=\frac{\gamma^{-n D}}{\pi^{2} G^{D-1}} .
$$

The height before deformation of the asperity is

$$
h_{\mathrm{n}}=z_{\mathrm{n}}(0)=\frac{G^{D-1}}{\gamma^{(2-D) n}} .
$$

In the loading process of asperity, the deformation will increase with the increase of normal contact load. Accordingly, asperity will change from elastic deformation to elastoplastic deformation and then to fully plastic deformation.

The elastic critical interference of asperity at initial yield is [16]

$$
\omega_{\text {nec }}=\left(\frac{3 \pi K H}{4 E^{\prime}}\right)^{2} R
$$

where $K=0.454+041 v, v$ is the Poisson ratio of the softer material, $H$ is the hardness of the softer material, $H=2.8 Y$, and $E^{\prime}$ is the equivalent elastic modulus, $\left(1 / E^{\prime}\right)=$ $\left(1-v_{1}^{\prime} / E_{1}\right)+\left(1-v_{2}^{\prime} / E_{2}\right) \cdot E_{1}$ and $E_{2}$ are, respectively, elastic modulus of two objects in contact with each other. $v_{1}^{\prime}$ and $v_{2}^{\prime}$ are, respectively, the corresponding Poisson ratios.

When $\omega_{n}<\omega_{\text {nec }}, a_{n}<a_{\text {nec }}$, the asperity is in a state of elastic deformation. According to Hertz theory, the contact area of the asperity is

$$
a_{\mathrm{n}}=\pi R \omega_{\mathrm{n}}
$$

Substituting equation (6) in equation (7), the critical contact area of the elastic asperity is

$$
a_{\mathrm{nec}}=\pi R \omega_{\mathrm{nec}}=\pi\left(\frac{3 \pi K H}{4 E^{\prime}}\right)^{2} R^{2}=\frac{1}{\pi}\left(\frac{3 K H \gamma^{-n D}}{4 G^{(D-1)} E^{\prime}}\right)^{2} .
$$

According to the Hertz contact theory, the normal load on a single asperity is

$$
f_{\text {ne }}=\frac{4}{3} E^{\prime} R_{n}^{1 / 2} \omega_{n}^{3 / 2} .
$$
obtain

$$
f_{\mathrm{ne}}=\frac{4 E^{\prime} a_{n}^{3 / 2} \pi^{1 / 2} G^{D-1}}{3 \gamma^{-D n}} .
$$

According to equations (6), (8), and (9), we can get the critical contact load of the elastic asperity:

$$
f_{\text {nec }}=K \cdot H \cdot a_{\text {nec }} \text {. }
$$

2.2. Elastic-Plastic Deformation of a Single Asperity. Literature [6] through the finite element analysis of a single asperity, it is concluded that when the asperity actual deformation is greater than the elastic critical interference $\left(\omega_{\mathrm{n}}>\omega_{\text {nec }}\right)$, the yield phenomenon begins to appear, and the elastoplastic deformation of the asperity occurs. According to the results of [6], the elastoplastic deformation of asperity can be divided into two different stages according to the ratio $\omega_{\mathrm{n}} / \omega_{\text {nec }}$, namely, the first elastoplastic deformation stage when $1<\omega_{\mathrm{n}} / \omega_{\text {nec }} \leq 6$ and the second elastoplastic deformation stage when $6<\omega_{n} / \omega_{\text {nec }} \leq 110$. Define $\omega_{\text {nepc }}=$ $6 \omega_{\text {nec }}$ as the first elastoplastic critical interference, where the actual contact area is $a_{\text {nepc }}$. The actual deformation of asperity $\omega_{\mathrm{npc}}=110 \omega_{\mathrm{nec}}$ is defined as the second elastoplastic critical interference, and the actual contact area is $a_{\mathrm{npc}}$. The relationship between contact area-deformation and contact load-deformation in the elastoplastic deformation stage of asperity is [6]

$$
\frac{a_{n}}{a_{\text {nec }}}=0.93\left(\frac{\omega_{n}}{\omega_{\text {nec }}}\right)^{1.136}
$$

$$
\begin{aligned}
\frac{f_{\text {nep } 1}}{f_{\text {nec }}}=1.03\left(\frac{\omega_{n}}{\omega_{\text {nec }}}\right)^{1.425}, \quad\left(\omega_{\text {nec }}<\omega_{\mathrm{n}} \leq 6 \omega_{\text {nec }}\right), \\
\frac{a_{\mathrm{n}}}{a_{\text {nec }}}=0.94\left(\frac{\omega_{\mathrm{n}}}{\omega_{\text {nec }}}\right)^{1.146}, \\
\frac{f_{\text {nep } 2}}{f_{\text {nec }}}=1.40\left(\frac{\omega_{\mathrm{n}}}{\omega_{\text {nec }}}\right)^{1.263}, \quad\left(6 \omega_{\text {nec }}<\omega_{\mathrm{n}} \leq 110 \omega_{\text {nec }}\right) .
\end{aligned}
$$

From the above equations, we can get 


$$
\begin{aligned}
a_{\mathrm{nepc}} & =7.1197 a_{\mathrm{nec}}, \\
f_{\mathrm{nep} 1} & =K H \times 1.1282 a_{\mathrm{nec}}^{-0.2544} a_{\mathrm{n}}^{1.2544}, \quad\left(a_{\mathrm{nec}}<a_{\mathrm{n}}<a_{\mathrm{nepc}}\right), \\
a_{\mathrm{npc}} & =205.3827 a_{\mathrm{nec}}, \\
f_{\mathrm{nep} 2} & =K H \times 1.4988 a_{\mathrm{nec}}^{-0.1021} a_{\mathrm{n}}^{1.1021}, \quad\left(a_{\mathrm{nepc}}<a_{\mathrm{n}}<a_{\mathrm{npc}}\right),
\end{aligned}
$$

where $f_{\text {nec }}$ is contact load for $\omega=\omega_{\text {nec }}$ and $f_{\text {nep1 }}$ and $f_{\text {nep2 }}$ are contact loads in the first elastoplastic stage and the second elastoplastic stage, respectively. Both $f_{\text {nep } 1}$ and $f_{\text {nep2 }}$ obtained above are related to the hardness $(H)$ of the material. However, according to the plastic strengthening principle, the hardness is not a constant when the material yields, but a function related to the deformation, that is, it changes with the deformation. Therefore, it is not accurate to describe elastoplastic deformation by the above formula. In order to express the characteristics of elastoplastic deformation more accurately, the concept of limit mean geometric hardness is introduced.

According to equations (13) and (14), $H_{\mathrm{G}}(a)$ is fitted into the following segmented relations.

The first elastoplastic deformation stage is

$$
H_{\mathrm{G} 1}\left(a_{\mathrm{n}}\right)=c_{1} Y\left(\frac{a_{\mathrm{n}}}{a_{\mathrm{nec}}}\right)^{c_{2}}, \quad\left(a_{\mathrm{nec}}<a_{\mathrm{n}} \leq a_{\mathrm{nepc}}\right) \text {. }
$$

The second elastoplastic deformation stage is

$$
H_{\mathrm{G} 2}\left(a_{\mathrm{n}}\right)=c_{3} Y\left(\frac{a_{\mathrm{n}}}{a_{\mathrm{nec}}}\right)^{c_{4}}, \quad\left(a_{\mathrm{nepc}}<a_{\mathrm{n}} \leq a_{\mathrm{npc}}\right) \text {, }
$$

where $c_{1}, c_{2}, c_{3}$, and $c_{4}$ are the coefficients to be solved.

(1) Equation (15) should satisfy two limiting conditions:

$$
\begin{gathered}
H_{\mathrm{G} 1}\left(a_{\mathrm{nec}}\right)=p_{\text {ea }}\left(a_{\mathrm{nec}}\right), \\
H_{\mathrm{G} 1}\left(a_{\text {nepc }}\right)=p_{\text {epal }}\left(a_{\text {nepc }}\right),
\end{gathered}
$$

where $p_{\mathrm{ea}}(a)$ is the average contact pressure of the asperity in elastic stage, which is given by $p_{\text {ea }}(a)=\left(f_{\text {ne }} / a\right) ; \quad p_{\text {epal }}(a)$ is the average contact pressure of the asperity in the first elastoplastic deformation stage and is given by $p_{\text {epal }}(a)=\left(f_{\text {nepl }} / a\right)$. Substituting equations (11) and (15) in equation (17), we can obtain

$$
\begin{aligned}
c_{1} Y & =K H, \\
c_{1} & =2.8 K .
\end{aligned}
$$

Substituting equations (13) and (15) in equation (18), we can obtain

$$
K H \times 1.1282 a_{\text {nec }}^{-0.2544} \times\left(7.1197 a_{\text {nec }}\right)^{0.2544}=2.8 K Y \times 7.1197^{c_{2}} .
$$

Derived from equation (21),

$$
c_{2}=\frac{\ln \left(1.1282 \times 7.1197^{0.2544}\right)}{\ln 7.1197} .
$$

Considering the change of hardness, the normal contact load of a single asperity in the first elastoplastic stage is

$$
f_{\text {nep } 1}^{\prime}=H_{\mathrm{G} 1}(a) \cdot a_{\mathrm{n}}
$$

Substituting equations (15), (20), and (22) in equation (23), new equations are yielded:

$$
f_{\text {nep } 1}^{\prime}=2.8 K Y a_{\text {nec }}^{-c_{2}} a_{\mathrm{n}}^{c_{2}+1} \text {. }
$$

(2) Equation (16) should satisfy two limiting conditions:

$$
\begin{gathered}
H_{\mathrm{G} 2}\left(a_{\text {nepc }}\right)=p_{\text {epa1 }}\left(a_{\text {nepc }}\right), \\
H_{\mathrm{G} 2}\left(a_{\mathrm{npc}}\right)=p_{\text {epa } 2}\left(a_{\mathrm{npc}}\right),
\end{gathered}
$$

where $p_{\text {epa2 }}(a)=\left(f_{\text {nep } 2} / a\right)$ is the average contact pressure of the asperity in the second elastoplastic stage. Substituting equations (13) and (16) in equation (25), we can obtain

$c_{3}(7.1197)^{c_{4}}=K \times 2.8 \times 1.1282 \times(7.1197)^{0.2544}$.

Substituting equations (14) and (16) in equation (26), we can obtain

$c_{3}(205.3827)^{c_{4}}=K \times 2.8 \times 1.4988 \times(205.3827)^{0.1021}$.

Simultaneous equations (27) and (28) obtained

$c_{4}=\frac{\ln 1.1282+0.2544 \ln 7.1197-\ln 1.4988-0.1021 \ln 205.3827}{\ln 7.1197-\ln 205.3827}$.

Substituting equation (29) in equation (27), we can obtain

$$
c_{3}=K \times 3.15896 \times(7.1197)^{0.2544-c_{4}} .
$$

Considering the change of hardness, the normal contact load of a single asperity in the second elastoplastic stage is

$$
\begin{aligned}
f_{\mathrm{nep} 2}^{\prime}= & H_{\mathrm{G} 2}(a) \cdot a_{\mathrm{n}}=K \times 3.15896 \times(7.1197)^{0.2544-c_{4}} Y \\
& \cdot a_{\mathrm{nec}}^{-c_{4}} \cdot a_{\mathrm{n}}^{c_{4}+1} .
\end{aligned}
$$

2.3. Full Plastic Deformation of a Single Asperity. As the deformation continues to increase, when $\omega_{\mathrm{n}}>110 \omega_{\text {nec }}$, the contact area $a_{\mathrm{n}}>a_{\mathrm{npc}}$ and the asperity enters the stage of full plastic deformation. At this stage, the hardness of the material is no longer affected by the deformation and can be regarded as a constant. When the hardness of the material is 
given, according to literature [7], the contact load and contact area of the asperity at this stage can be expressed as

$$
\begin{aligned}
f_{\mathrm{np}} & =H a_{\mathrm{n}}, \\
a_{\mathrm{n}} & =2 \pi R_{\mathrm{n}} \omega_{\mathrm{n}} .
\end{aligned}
$$

In conclusion, with the increase of load and deformation, the contact area of the same asperity increases gradually, i.e., $a_{\text {nec }}<a_{\text {nepc }}<a_{\text {npc }}$. With the increase of the load and contact area, the asperity underwent elastic deformation, first elastoplastic deformation, second elastoplastic deformation, and full plastic deformation successively. Under constant load and deformation, the actual contact area of the asperity is related to the radius of curvature at the vertex of the asperity.

2.4. Asperity's Frequency Exponent $n$. When using W-M function to describe the surface profile of an asperity, the profile function is related to the asperity's frequency exponent. In other words, the radius of curvature at the vertex of the asperity and the height of the asperity vary with the frequency exponent when the load is constant. According to the equations (5)-(7), it was found that the value of $h_{\mathrm{n}}, R_{\mathrm{n}}$, and $\omega_{\text {nec }}$ correlated with the frequency exponent. When the frequency exponent is constant, the deformation of the asperity is not greater than the height of the asperity under the action of the load. In order to obtain the critical value of the frequency exponent, we take $h_{\mathrm{n}}=\omega_{\text {nec }}$, i.e., $\left(G^{D-1} / \gamma^{(2-D) n_{\mathrm{ec}}}\right)=\left(3 K H / 4 E^{\prime}\right)^{2} \cdot\left(\gamma^{-n_{\mathrm{ec}} D} / G^{D-1}\right)$.

The elastic critical frequency exponent can be obtained as follows:

$$
n_{\mathrm{ec}}=\operatorname{int}\left\{\frac{\ln \left[\left(3 K H / 4 E^{\prime}\right)^{2} \cdot G^{2(1-D)}\right]}{2(D-1) \ln \gamma}\right\},
$$

where int \{\} is the integer part of the value in the parenthesis.

Similarly, the first elastoplastic critical frequency exponent can be obtained:

$$
n_{\mathrm{epc}}=\operatorname{int}\left\{\frac{\ln \left[6\left(3 K H / 4 E^{\prime}\right)^{2} \cdot G^{2(1-D)}\right]}{2(D-1) \ln \gamma}\right\} .
$$

The second elastoplastic critical frequency exponent can be obtained:

$$
n_{\mathrm{pc}}=\operatorname{int}\left\{\frac{\ln \left[110\left(3 K H / 4 E^{\prime}\right)^{2} \cdot G^{2(1-D)}\right]}{2(D-1) \ln \gamma}\right\} .
$$

From the above, when the asperity frequency exponent is $n_{\text {min }}<n \leq n_{\text {ec }}$, elastic deformation only takes place in these asperities under contact load. When $n_{\mathrm{ec}}<n \leq n_{\mathrm{epc}}$, elastic deformation or the first elastoplastic deformation can take place in these asperities. When $n_{\mathrm{epc}}<n \leq n_{\mathrm{pc}}$, elastic deformation, the first elastoplastic deformation, or the second elastoplastic deformation can take place in these asperities, and full plastic deformation never occur. When $n_{\mathrm{pc}}<n \leq n_{\max }$, elastic deformation, elastoplastic deformation, or full plastic deformation can take place in these asperities.

\section{Actual Contact Area and Normal Contact Load of Joint Interface}

According to reference [10], when the asperity frequency exponent is $n$, the area distribution density function of the asperity on the joint interface is defined as

$$
n_{\mathrm{n}}(a)=\frac{1}{2} D \cdot \frac{a_{\mathrm{nl}}^{D / 2}}{a^{(D+2) / 2}}, \quad\left(0<a \leq a_{\mathrm{nl}}, 1<D<2\right),
$$

where $a_{\mathrm{nl}}$ represents the largest contact area when the asperity's frequency exponent is $n$.

In order to simplify equation (36), we define the area distribution function of the asperity of any frequency exponent as $n_{\mathrm{n}}(a)=\operatorname{Mn}(a)$. According to reference [17], the actual contact area of joint interface is

$$
A_{\mathrm{r}}=\sum_{n=n_{\min }}^{n_{\max }} \int_{0}^{a_{\mathrm{nl}}} n_{\mathrm{n}}(a) a d a=M \sum_{n=n_{\min }}^{n_{\max }} \int_{0}^{a_{\mathrm{nl}}} n(a) a d a,
$$

where $M=\left(a_{1} / \sum_{n=n_{\min }}^{n_{\max }} a_{\mathrm{nl}}\right)\left(n_{\min } \leq n \leq n_{\max }, a_{1}=\max \left\{a_{\mathrm{nl}}\right\}\right)$.

3.1. When the Frequency Exponent Belongs to $n_{\min }<n \leq n_{e c}$. When the frequency exponent belongs to $n_{\min }<n \leq n_{\mathrm{ec}}$, even if these asperities are completely deformed, only elastic deformation will occur, and $a_{\mathrm{nl}}<a_{\text {nec }}$. In this case, the actual contact area of the joint interface is defined as $A_{\mathrm{r} 1}$ :

$$
A_{\mathrm{r} 1}=\sum_{n=n_{\min }}^{n_{\mathrm{ec}}} \int_{0}^{a_{\mathrm{nl}}} M n(a) a d a=\frac{M D}{2-D} \sum_{n=n_{\min }}^{n_{\mathrm{ec}}} a_{\mathrm{nl}} .
$$

In this case, the contact load of the joint interface is as follows:

$$
F_{\mathrm{r} 1}=\sum_{n=n_{\min }}^{n_{\mathrm{ec}}} \int_{0}^{a_{\mathrm{nl}}} f_{\mathrm{ne}} M n(a) d a .
$$

Substituting equation (11) in equation (39), we can obtain

$$
F_{\mathrm{r} 1}=\frac{M D}{3-D} \sum_{n=n_{\min }}^{n_{\mathrm{ec}}} \frac{4 E \pi^{1 / 2} G^{(D-1)}}{3 \gamma^{-D n}} a_{\mathrm{nl}}^{3 / 2}
$$

3.2. When the Frequency Exponent Belongs to $n_{e c}<n \leq n_{e p c}$. When the frequency exponent belongs to $n_{\mathrm{ec}}<n \leq n_{\mathrm{epc}}$, for the case $a_{\text {nec }}<a_{\text {nl }} \leq a_{\text {nepc }}$, elastic deformation or the first elastoplastic deformation may take place in these asperities. At this point, the actual contact area of the joint interface consists of two parts, the elastic deformation stage and the first elastoplastic deformation stage: 


$$
\begin{aligned}
& A_{\mathrm{r} 2}=A_{\mathrm{re}}+A_{\mathrm{rep} 1}, \\
& A_{\mathrm{re}}=\sum_{n=n_{\mathrm{ec}}+1}^{n_{\mathrm{epc}}} \int_{0}^{a_{\mathrm{nec}}} M n(a) a d a=\frac{M D}{2-D} \sum_{n=n_{\mathrm{ec}}+1}^{n_{\mathrm{epc}}} a_{\mathrm{nec}}^{(2-D) / 2} a_{\mathrm{nl}}^{D / 2} .
\end{aligned}
$$

For the determined frequency exponent, the maximum actual contact area of the asperity appears at the maximum deformation amount $\omega_{n}$, where the maximum value of the elastic deformation phase $\omega_{\mathrm{n}}$ appears at $\omega_{\text {nec }}$, whereupon formula (42) is simplified to

$$
\begin{aligned}
A_{\mathrm{re}}= & \frac{M D}{2-D} \sum_{n=n_{\mathrm{ec}}+1}^{n_{\mathrm{epc}}} a_{\mathrm{nec}}=\frac{M D}{(2-D) \pi} \sum_{n=n_{\mathrm{ec}}+1}^{n_{\mathrm{epc}}}\left(\frac{3 K H \gamma^{-D n}}{4 G^{(D-1)} E^{\prime}}\right)^{2}, \\
A_{\mathrm{rep} 1}= & \sum_{n=n_{\mathrm{ec}}+1}^{n_{\mathrm{epc}}} \int_{a_{\mathrm{nec}}}^{a_{\mathrm{nl}}} M n(a) a d a=\frac{M D}{2-D} \\
& \cdot \sum_{n=n_{\mathrm{ec}}+1}^{n_{\mathrm{epc}}}\left[a_{\mathrm{nl}}^{(2-D) / 2}-a_{\mathrm{nec}}^{(2-D) / 2}\right] a_{\mathrm{nl}}^{D / 2} .
\end{aligned}
$$

The contact load is given by

$$
\begin{aligned}
F_{\mathrm{r} 2}= & F_{\mathrm{re}}+F_{\text {rep } 1} \\
F_{\mathrm{re}}= & \sum_{n=n_{\mathrm{ec}}+1}^{n_{\mathrm{epc}}} \int_{0}^{a_{\mathrm{nec}}} f_{\mathrm{ne}} M n(a) d a \frac{9 M D(K H)^{3}}{16(3-D)\left(E^{\prime} \pi G^{D-1}\right)^{2}} \\
& \cdot \sum_{n=n_{\mathrm{ec}}+1}^{n_{\mathrm{epc}}} \gamma^{-2 D n},
\end{aligned}
$$$$
F_{\text {rep1 }}=\sum_{n=n_{\mathrm{ec}}+1}^{n_{\mathrm{epc}}} \int_{a_{\mathrm{nec}}}^{a_{n l}} f_{\mathrm{nep} 1}^{\prime} M n(a) d a .
$$

Substituting equations (24) and (36) in equation (46), we can obtain

$$
F_{\text {rep } 1}=\frac{2.8 K Y M D}{2 c_{2}-D+2} \sum_{n=n_{\mathrm{ec}}+1}^{n_{\mathrm{epc}}}\left[a_{\mathrm{nec}}^{-c_{2}} a_{\mathrm{nl}}^{c_{2}+1}-a_{\mathrm{nec}}^{(2-D) / 2} a_{\mathrm{nl}}^{D / 2}\right]
$$

3.3. When the Frequency Exponent Belongs to $n_{e p c}<n \leq n_{p c}$. When the frequency exponent belongs to $n_{\mathrm{epc}}<n \leq n_{\mathrm{pc}}$, for the case $a_{\text {nepc }}<a_{\mathrm{nl}} \leq a_{\mathrm{npc}}$, elastic deformation, the first elastoplastic deformation, or the second elastoplastic deformation may take place in these asperities. At this point, the actual contact area of the joint interface consists of three parts: the elastic deformation stage, the first elastoplastic deformation stage, and the second elastoplastic deformation stage:

$$
\begin{aligned}
A_{\mathrm{r} 3}= & A_{\mathrm{re}}+A_{\mathrm{rep} 1}+A_{\mathrm{rep} 2}, \\
A_{\mathrm{re}}= & \sum_{n=n_{\mathrm{epc}}+1}^{n_{\mathrm{pc}}} \int_{0}^{a_{\mathrm{nec}}} M n(a) a d a=\frac{M D}{(2-D) \pi} \\
& \cdot \sum_{n=n_{\mathrm{epc}}+1}^{n_{\mathrm{pc}}}\left(\frac{3 K H \gamma^{-D n}}{4 G^{D-1} E^{\prime}}\right)^{2}, \\
A_{\mathrm{rep} 1}= & \sum_{n=n_{\mathrm{epc}}+1}^{n_{\mathrm{pc}}} \int_{a_{\mathrm{nec}}}^{a_{\mathrm{nepc}}} M n(a) a d a \\
= & \frac{M D}{\pi(2-D)}\left(7.1197-7.1197^{D / 2}\right) \sum_{n=n_{\mathrm{epc}}+1}^{n_{\mathrm{pc}}}\left(\frac{3 K H \gamma^{-D n}}{4 G^{D-1} E^{\prime}}\right)^{2}, \\
A_{\mathrm{rep} 2}= & \sum_{n=n_{\mathrm{epc}}+1}^{n_{\mathrm{pc}}} \int_{a_{\mathrm{nepc}}}^{a_{\mathrm{nl}}} M n(a) a d a \\
(2-D) & \sum_{n=n_{\mathrm{epc}}+1}^{n_{\mathrm{pc}}}\left[a_{n l}^{(2-D) / 2}-\left(7.1197 a_{\mathrm{nec}}\right)^{(2-D) / 2}\right] a_{\mathrm{nl}}^{D / 2} . \\
& M D
\end{aligned}
$$

In this case, the contact load of the joint interface is as follows:

$$
\begin{aligned}
F_{\mathrm{r} 3}= & F_{\mathrm{re}}+F_{\mathrm{rep} 1}+F_{\mathrm{rep} 2}, \\
F_{\mathrm{re}}= & \sum_{n=n_{\mathrm{epc}}+1}^{n_{\mathrm{pc}}} \int_{0}^{a_{\mathrm{nec}}} f_{\mathrm{ne}} M n(a) d a \\
= & \frac{9 M D(K H)^{3}}{16(3-D)\left(E^{\prime} \pi G^{D-1}\right)^{2}} \sum_{n=n_{\mathrm{epc}}+1}^{n_{\mathrm{pc}}} \gamma^{-2 D n}, \\
F_{\mathrm{rep} 1}= & \frac{2.8 K Y M D}{2 c_{2}-D+2}\left(7.1197^{c_{2}+1}-7.1197^{D / 2}\right) \\
& \cdot \sum_{n=n_{\mathrm{epc}}+1}^{n_{\mathrm{pc}}} \frac{1}{\pi}\left(\frac{3 K H \gamma^{-D n}}{4 G^{D-1} E^{\prime}}\right)^{2} .
\end{aligned}
$$

When the second elastoplastic deformation occurs, the normal contact load of the joint interface is as follows: 


$$
F_{\text {rep2 }}=\sum_{n=n_{\text {epc }}+1}^{n_{\mathrm{pc}}} \int_{a_{\text {nepc }}}^{a_{\text {nl }}} f_{\text {nep2 }}^{\prime} M n(a) d a .
$$

Substituting equations (31) and (38) in equation (46), we can obtain

$$
\begin{aligned}
F_{\text {rep } 2}= & \frac{2.106 \times 7.1197^{0.2544-c_{4}} K Y M D}{2 c_{4}-D+2} \\
& \cdot \sum_{n=n_{\mathrm{epc}}+1}^{n_{\mathrm{pc}}}\left(a_{\mathrm{nec}}^{-c_{4}} a_{\mathrm{nl}}^{c_{4}+1}-7.1197^{c_{4}-(D / 2)+1} a_{\mathrm{nec}}^{1-(D / 2)} a_{\mathrm{nl}}^{(\mathrm{D} / 2)}\right) .
\end{aligned}
$$

3.4. When the Frequency Exponent Belongs to $n_{p c}<n$. When the frequency exponent belongs to $n_{\mathrm{pc}}<n$, elastic deformation, elastoplastic deformation, or full plastic deformation may take place in these asperities. The actual contact area of the joint interface can be evaluated as

$$
\begin{aligned}
& A_{\mathrm{r} 4}=A_{\mathrm{re}}+A_{\mathrm{rep} 1}+A_{\mathrm{rep} 2}+A_{\mathrm{rp}}, \\
& A_{\mathrm{re}}=\sum_{n=n_{\mathrm{pc}}+1}^{n_{\max }} \int_{0}^{a_{\mathrm{nec}}} M n(a) a d a=\frac{M D}{2-D} \sum_{n=n_{\mathrm{pc}}+1}^{n_{\max }} a_{\mathrm{nec}}, \\
& A_{\mathrm{rep} 1}=\sum_{n=n_{\mathrm{pc}}+1}^{n_{\max }} \int_{a_{\mathrm{nec}}}^{a_{\mathrm{nepc}}} M n(a) a d a \\
&=\frac{M D}{2-D}\left(7.1197-7.1197^{D / 2}\right) \sum_{n=n_{\mathrm{pc}}+1}^{n_{\max }} a_{\mathrm{nec}}, \\
& A_{\mathrm{rep} 2}=\sum_{n=n_{\mathrm{pc}}+1}^{n_{\max }} \int_{a_{\mathrm{nepc}}}^{a_{\mathrm{npc}}} M n(a) a d a \\
&=\frac{M D}{2-D}\left(205.3827-7.1197^{1-(D / 2)} \cdot 205.3827^{D / 2}\right) \sum_{n=n_{\mathrm{pc}}+1}^{n_{\max }} a_{\mathrm{nec}}, \\
& A_{\mathrm{rp}}=\sum_{n=n_{\mathrm{pc}}+1}^{n_{\max }} \int_{a_{\mathrm{npc}}}^{a_{n l}} M n(a) a d a \\
&=\frac{M D}{2-D} \sum_{n=n_{\mathrm{pc}}+1}^{n_{\max }}\left[a_{n l}^{(2-D) / 2}-\left(205.3827 a_{\mathrm{nec}}\right)^{(2-D) / 2}\right] a_{n l}^{D / 2} \cdot \\
& \mathrm{or}
\end{aligned}
$$

In this case, the contact load of the joint interface is as follows:

$$
\begin{aligned}
& F_{\mathrm{r} 4}=F_{\mathrm{re}}+F_{\mathrm{rep} 1}+F_{\mathrm{rep} 2}+F_{\mathrm{rp}} \text {, } \\
& F_{\mathrm{re}}=\sum_{n=n_{\mathrm{pc}}+1}^{n_{\max }} \int_{0}^{a_{\text {nec }}} f_{\mathrm{ne}} M n(a) d a=\frac{M D K H}{(3-D) \pi} \sum_{n=n_{\mathrm{pc}}+1}^{n_{\max }} a_{\mathrm{nec}}, \\
& F_{\text {rep } 1}=\sum_{n=n_{\text {pc }}+1}^{n_{\text {max }}} \int_{a_{\text {nec }}}^{a_{\text {nepc }}} f_{\text {nep1 }}^{\prime} M n(a) d a \\
& =\frac{2.8 K Y M D}{2 c_{2}-D+2}\left(7.1197^{c_{2}+1}-7.1197^{D / 2}\right) \sum_{n=n_{\mathrm{pc}}+1}^{n_{\max }} a_{\text {nec }}, \\
& F_{\text {rep2 }}=\frac{2.106 \times 7.1197^{0.2544-c_{4}} K Y M D}{2 c_{4}-D+2} \\
& \cdot \sum_{n=n_{\mathrm{pc}}+1}^{n_{\max }}\left(a_{\mathrm{nec}}^{-c_{4}} a_{\mathrm{nl}}^{c_{4}+1}-7.1197^{c_{4}-(D / 2)+1} a_{\mathrm{nec}}^{1-(D / 2)} a_{\mathrm{nl}}^{D / 2}\right) \\
& =\frac{2.106 \times 7.1197^{0.2544-c_{4}} K Y M D}{2 c_{4}-D+2} \\
& \cdot\left(205.3827^{c_{4}+1}-205.3827^{D / 2} \cdot 7.1197^{c_{4}-(D / 2)+1}\right) \\
& \cdot \sum_{n=n_{\mathrm{pc}}+1}^{n_{\max }} a_{\mathrm{nec}}, \\
& F_{\mathrm{rp}}=\sum_{n=n_{\mathrm{pc}}+1}^{n_{\max }} \int_{a_{\mathrm{npc}}}^{a_{n l}} f_{\mathrm{np}} M n(a) d a \\
& =\frac{M H D}{2-D} \sum_{n=n_{\mathrm{pc}}+1}^{n_{\mathrm{max}}} a_{\mathrm{nl}}^{D / 2}\left[a_{\mathrm{nl}}^{1-(D / 2)}-\left(205.3827 a_{\mathrm{nec}}\right)^{1-(D / 2)}\right] .
\end{aligned}
$$

For all frequency exponents, the total actual contact area of the joint interface is

$$
A_{\mathrm{r}}=A_{\mathrm{r} 1}+A_{\mathrm{r} 2}+A_{\mathrm{r} 3}+A_{\mathrm{r} 4} .
$$

The total contact load of the joint interface is

$$
F_{\mathrm{r}}=F_{\mathrm{r} 1}+F_{\mathrm{r} 2}+F_{\mathrm{r} 3}+F_{\mathrm{r} 4} \text {. }
$$

The total real contact area and the total contact load in a nondimensional form can be written as follows:

$$
\begin{aligned}
& A_{\mathrm{r}}^{*}=\frac{A_{\mathrm{r}}}{A_{\mathrm{a}}}, \\
& F_{\mathrm{r}}^{*}=\frac{F_{\mathrm{r}}}{A_{\mathrm{a}} E},
\end{aligned}
$$

where $A_{\mathrm{a}}$ is the nominal contact area and is given by $A_{\mathrm{a}}=L^{2}$, $L=1 / \gamma^{n_{\min }}$. 


\section{Results Analysis}

In order to further analyze the above calculation results, the parameters of equivalent joint interface are taken as shown in Table 1 [16].

Figure 2 shows the relation between all critical contact areas and frequency exponents of single asperity when $D=1.5$. It can be seen from the figure that as for one definite asperity, when frequency exponent $n$ is certain, elastic critical contact area is minimum, followed by the first elastoplastic critical contact area, and the second elastoplastic critical contact area is maximum. With gradual increase of contact load, the contact area increases. The single asperity is firstly subject to elastic deformation, followed by the first elastoplastic deformation, the second elastoplastic deformation, and fully plastic deformation successively, which is consistent with typical contact mechanics theory. As for different asperities, with increase of frequency exponent, all critical contact areas decrease correspondingly, which shows that elastic critical contact area, the first elastoplastic critical contact area, and the second elastoplastic critical contact area are all related to frequency exponent $n$.

Figure 3 shows the relation curve between fractal dimension $D$ and critical frequency exponent $n$ of asperity. When fractal dimension is definite, elastic critical frequency exponent $n_{\mathrm{ec}}$, the first elastoplastic critical frequency exponent $n_{\text {epc }}$, and the second elastoplastic critical frequency exponent $n_{\mathrm{pc}}$ increase gradually. As shown in Figure 3, when $D<1.06, n_{\mathrm{ec}}, n_{\mathrm{epc}}$, and $n_{\mathrm{pc}}$ are all negative. As for asperities with minimum value of frequency exponent being 0 , elastic deformation, elastoplastic deformation, and fully plastic deformation will all occur. When $D=1.13, n_{\mathrm{ec}}$ and $n_{\mathrm{epc}}$ are negative and $n_{\mathrm{pc}}$ is positive. At this time, as for asperities with minimum value of frequency exponent being 0 , elastic deformation, elastoplastic deformation will occur, except fully plastic deformation.

For $D=1.5, G=2.5 \times 10^{-9} \mathrm{~m}, H=5.5 \times 10^{9} \mathrm{~N} / \mathrm{m}^{2}$, we can obtain the elastic critical frequency exponent $n_{\mathrm{ec}}=32$, the first elastoplastic critical frequency exponent $n_{\mathrm{epc}}=36$, and the second elastoplastic critical frequency exponent $n_{\mathrm{pc}}=43$. These asperities whose frequency exponents range from 20 to 32 are only under elastic deformation. Elastic deformation and the first elastoplastic deformation can occur in these asperities whose frequency exponents range from 33 to 36 . Elastic deformation, the first elastoplastic deformation, and the second elastoplastic deformation can occur in these asperities whose frequency exponents range from 37 to 43 . When frequency exponents range from 43 to 50 , all deformations types can occur in these asperities.

Figure 4 shows relation comparison diagram between contact load and contact area of single asperity with and without hardness change at the first elastoplastic stage. The comparison diagram is simulation result when $n=33$. It can be seen from the figure that with gradual increase of contact area, with contact area of single asperity over $3.2 \times 10^{-13} \mathrm{~m}^{2}$, contact load of the same asperity with hardness change will be less than that without hardness change. In addition, as the amount of deformation increases, the difference between them tends to increase.
TABLE 1: The parameters of equivalent joint interface.

\begin{tabular}{lc}
\hline Parameters & Values \\
\hline Equivalent elastic modulus $E^{\prime}$ & $7.2 \times 10^{10} \mathrm{~N} / \mathrm{m}^{2}$ \\
Poisson's ratio $v$ & 0.17 \\
Initial hardness $H$ & $5.5 \times 10^{9} \mathrm{~N} / \mathrm{m}^{2}$ \\
Profile scale parameter $G$ & $2.5 \times 10^{-9} \mathrm{~m}$ \\
Fractal dimension $D$ & $1<D<2$ \\
Frequency exponent $n$ & $20 \sim 50$ \\
\hline
\end{tabular}

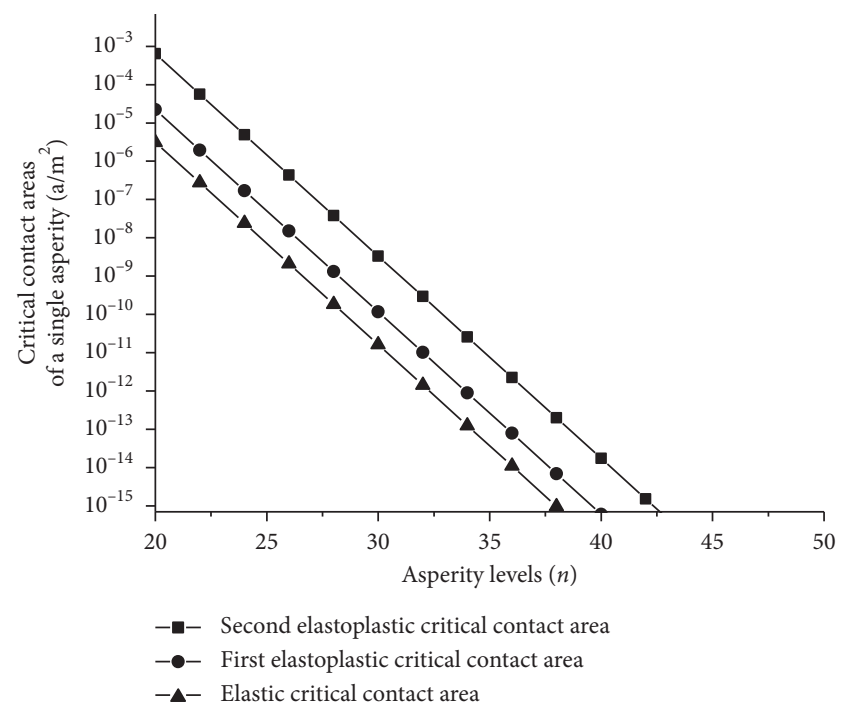

Figure 2: The relationship between critical contact area and frequency exponent of a single asperity.

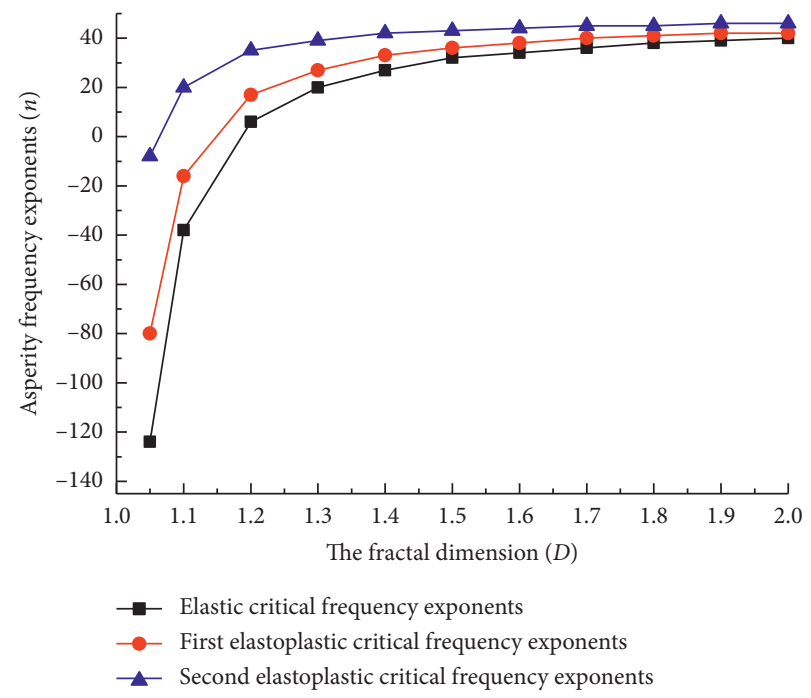

FIgURE 3: The relationship between fractal dimension $D$ and critical frequency exponent $n$ of a single asperity.

Figure 5 shows relation comparison diagram between contact load and contact area of single asperity with and without hardness change at the second elastoplastic stage. The comparison diagram is simulation result when $n=37$. It can be seen from the figure that when the deformation is 


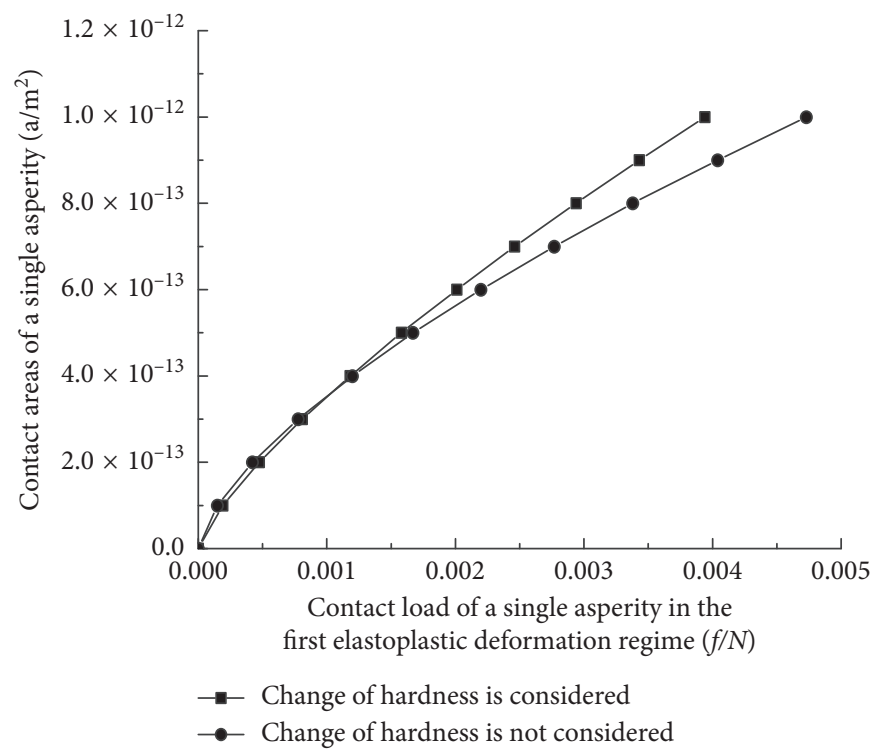

FIGURE 4: The relationship between contact load and contact area of single asperity in the first elastoplastic deformation stage.

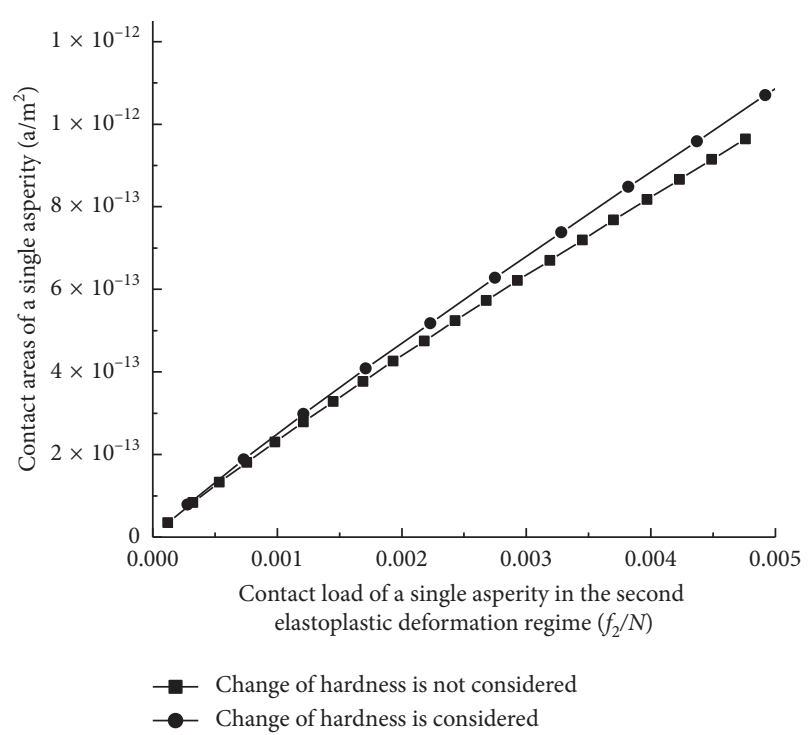

FIGURE 5: The relationship between contact load and contact area of single asperity in the second elastoplastic deformation stage.

definite, contact load of the same asperity with hardness change will be less than that without hardness change. In addition, with increase of deformation amount, the difference between them tends to increase, which is consistent with the change trend at the first elastoplastic stage.

Figure 6 shows the relation between limit mean geometric hardness and contact area (logarithm) of single asperity at the first elastoplastic deformation stage. Figure 6(a) shows relation curve that $D=1.1,1.3,1.5,1.7$ when $n=34$. Figure 6(b) shows relation curve that $n=32,33,34,35$ when $D=1.5$. It can be seen from Figure 6 that the limit mean geometric hardness of single asperity is related to contact area, fractal dimension, and frequency exponent in the first elastoplastic deformation stage. The limit mean geometric hardness increases with increase of contact area. When $n$ is definite, the relation between limit mean geometric hardness and contact area of asperity is related to fractal dimension $D$. The larger the $D$ is, the more obvious the relation curve between them changes; when $D$ is definite, the relation between limit mean geometric hardness and contact area of asperity is related to frequency exponent $n$. The smaller $n$ is, the more obvious the relation curve between them changes.

Figure 7 shows the relation between limit mean geometric hardness and contact area (logarithm) of single asperity at the second elastoplastic deformation stage. Figure 7 (a) shows the relation curve $n=40$ that $D=1.1,1.3$, $1.5,1.7$ when $n=40$. Figure $7(\mathrm{~b})$ shows the relation curve that $n=36,38,40,42$ when $D=1.5$.

During loading, the contact area increases with the increase of deformation of a single asperity. The ratio of deformation to the natural height of the asperity is defined as the pushing coefficient, namely, the pushing coefficient $k=\omega_{\mathrm{n}} / h_{\mathrm{n}}, 0 \leq k \leq 0.9$. When fractal dimension is 1.5 , we will research the relation between contact load and contact area of single asperity with frequency exponent $n$ being 30, 35, and 40 , respectively, during loading.

When $n=30$, the asperity will only be subject to elastic deformation. During loading, even the pushing coefficient $k$ is maximum, no plastic deformation will occur. The relation between contact area and contact load is $f \sim a^{1.5}$ approximately, as shown in Figure 8(a).

As is shown in Figure 8(b), when $n=35$, elastic deformation and the first elastoplastic deformation may take place in the asperity during loading. When the pushing coefficient $k$ is less than 0.247 , the asperity will under elastic deformation. At this time, the relation between contact area and contact load is $f \sim a^{1.5}$ approximately; when the pushing coefficient is over 0.247 , the first elastoplastic deformation occurs. At this time, the relation between contact area and contact load is $f \sim a^{1.1093}$ approximately. As is shown in Figure $8(\mathrm{c})$, when $n=40$, elastic deformation, the first elastoplastic deformation, and the second elastoplastic 


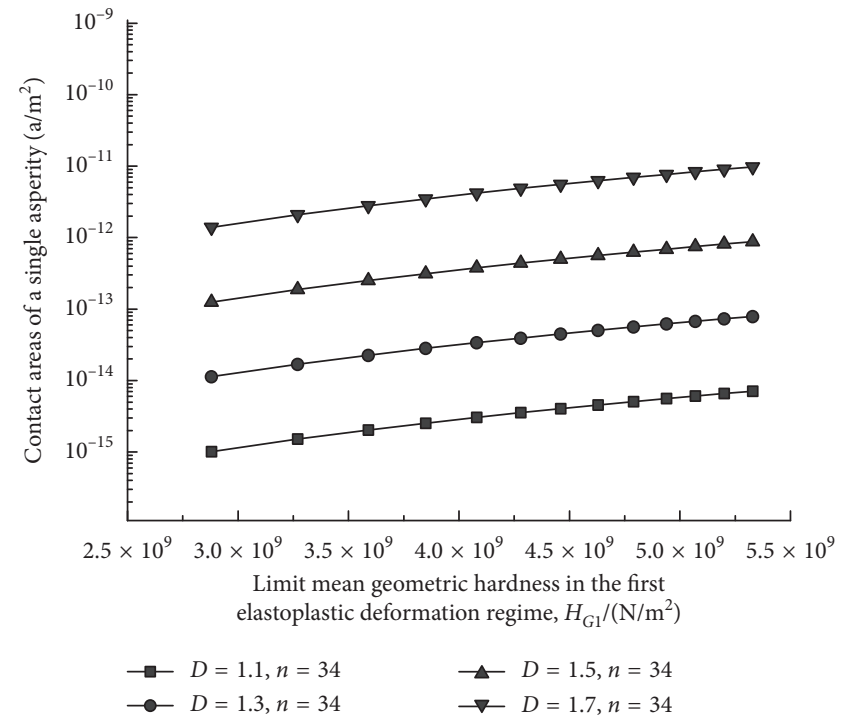

(a)

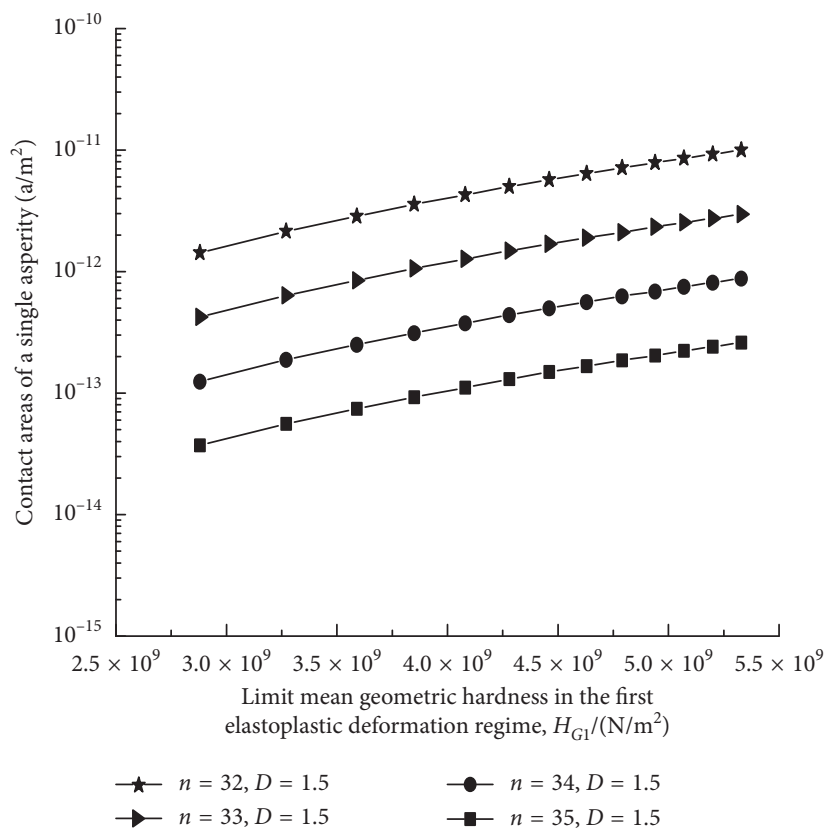

(b)

FIGURE 6: The relationship between limit mean geometric hardness and contact for single asperity during the first elastoplastic deformation stage. (a) $n=34,1.1 \leq D \leq 1.7$. (b) $D=1.5,32 \leq n \leq 35$.

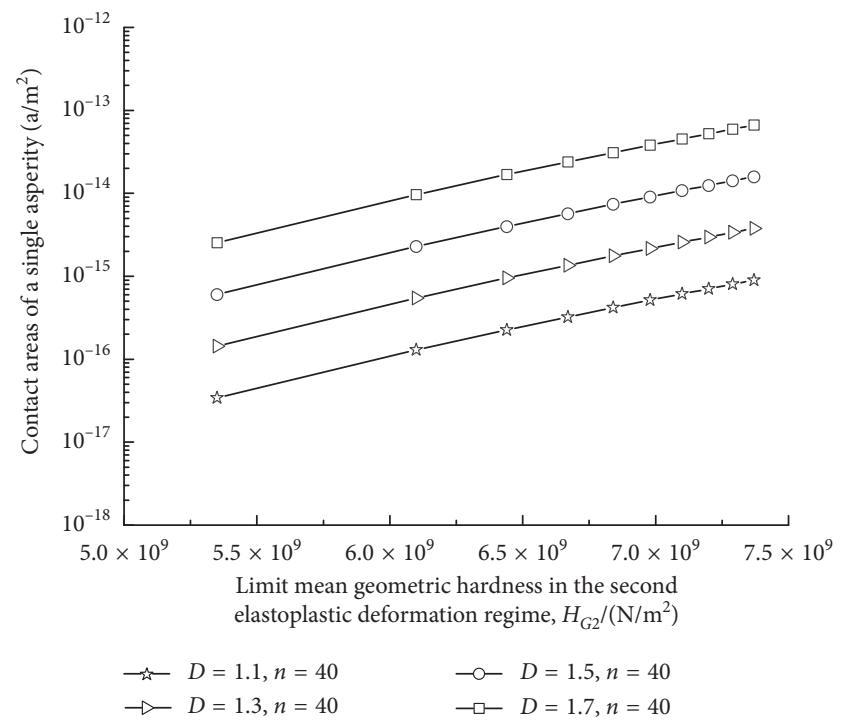

(a)

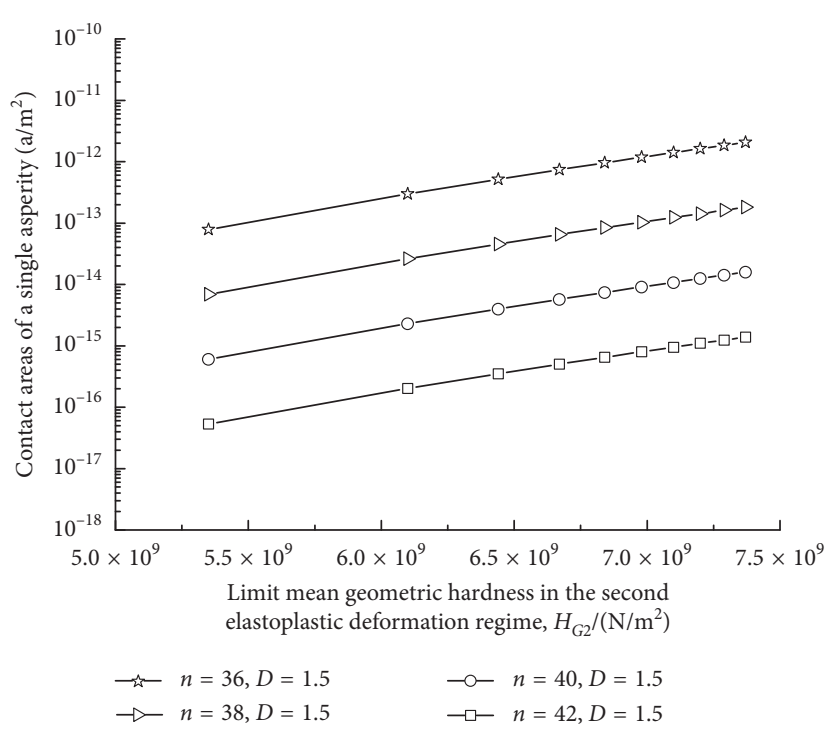

(b)

Figure 7: The relationship between limit mean geometric hardness and contact for single asperity during the second elastoplastic deformation stage. (a) $n=40,1.1 \leq D \leq 1.7$. (b) $D=1.5,36 \leq n \leq 42$.

deformation may take place in the asperity during loading. When the pushing coefficient is greater than 0.1954, the asperity begins to enter the second elastoplastic deformation, the relation between contact area and contact load is $f \sim a^{1.0977}$ approximately. When $n=45$ and the pushing coefficient is greater than 0.472 , the asperity begins to enter fully plastic deformation, the relation between contact area and contact load is $f \sim a$ approximately.
Figure 9 shows that when the minimum frequency exponent is 20 and the maximum value is 32 , the actual contact area of the joint interface increases with the increase of the total contact load, and the relation between them is $F_{r}^{*} \sim A_{r}^{* 1.5}$ approximately. During the whole deformation process, the joint interface appears to be of elastic property.

Figure 10 shows the relation between dimensionless total real contact area and the dimensionless total contact loading 


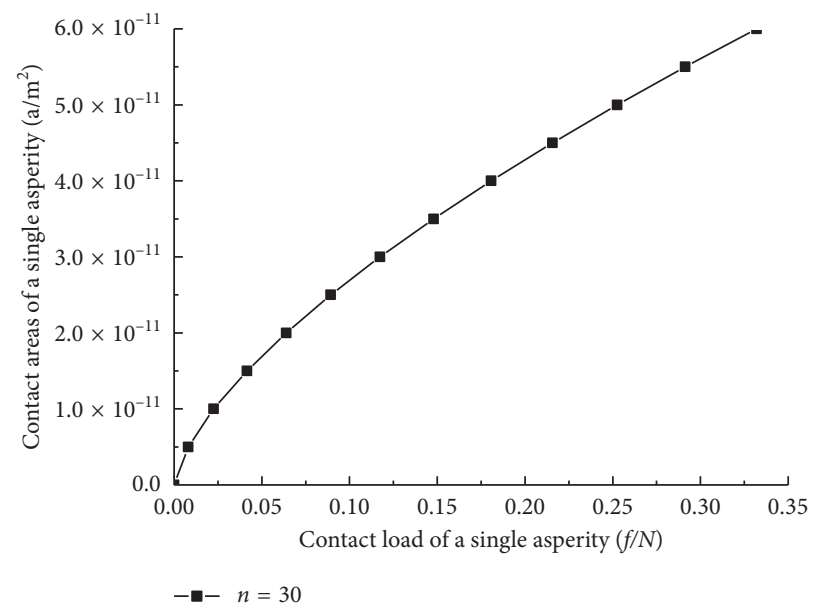

(a)

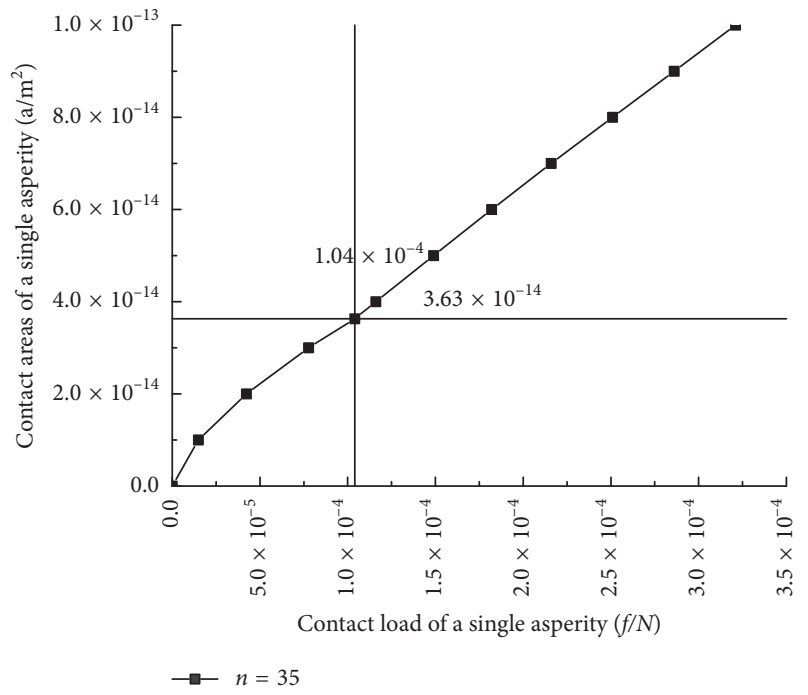

(b)

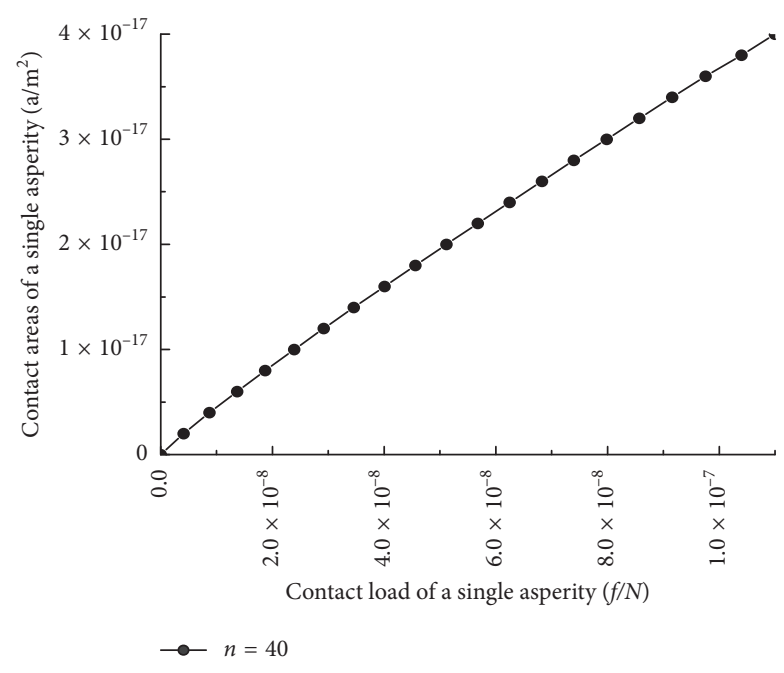

(c)

FIGURE 8: The relationship between contact load and contact area of a single asperity during loading.

of the joint interface when minimum value and maximum value of frequency exponent of asperities are, respectively, 20 and 36 . When the pushing coefficient $k$ is 0.1648 , the asperity begins to be subject to the first elastoplastic deformation, and the relation between dimensionless total real contact area and the dimensionless total contact loading is $F_{r}^{*} \sim A_{r}^{* 1.5}$ approximately. The joint interface appears to be of elastic property. As the load continues increasing, when the pushing coefficient $k$ is 0.5564 ; at this point, $F_{r}^{*}>6.9023 \times 10^{-3}$, the relation between above load and area is approximately $F_{r}^{*} \sim A_{r}^{* 1.1093}$. The joint interface presents elastoplastic properties. At this time, the first elastoplastic deformation takes place in these asperities whose frequency exponents range $33 \sim 36$.

Figure 11 shows the relation between dimensionless total real contact area and the dimensionless total contact loading of the joint interface when minimum value and maximum value of frequency exponent of asperities are, respectively, 44 and 50 . When the pushing coefficient $k$ is 0.0621 , the asperity begins to be subject to the second elastoplastic deformation, at this point, $0.0016<F_{\mathrm{r}}^{*}<0.023$, and the relation between dimensionless total real contact area and the dimensionless total contact loading is $F_{\mathrm{r}}^{*} \sim A_{\mathrm{r}}^{* 1.0977}$ approximately. When the pushing coefficient $k$ is 0.7076 , the asperity begins to be subject to the fully plastic deformation; at this point, $F_{\mathrm{r}}^{*}>0.023$, and the relation between dimensionless total real contact area and the dimensionless total contact loading is approximately $F_{r}^{*} \sim A_{r}^{*}$.

\section{Conclusions}

(1) The contact mechanical properties of a single asperity on a joint interface are related to the frequency exponent of the asperity, while the frequency exponent of an asperity is related to the fractal dimension and the profile scale parameter. In this paper, the critical frequency exponents of each deformation stage of a single asperity are obtained, and 


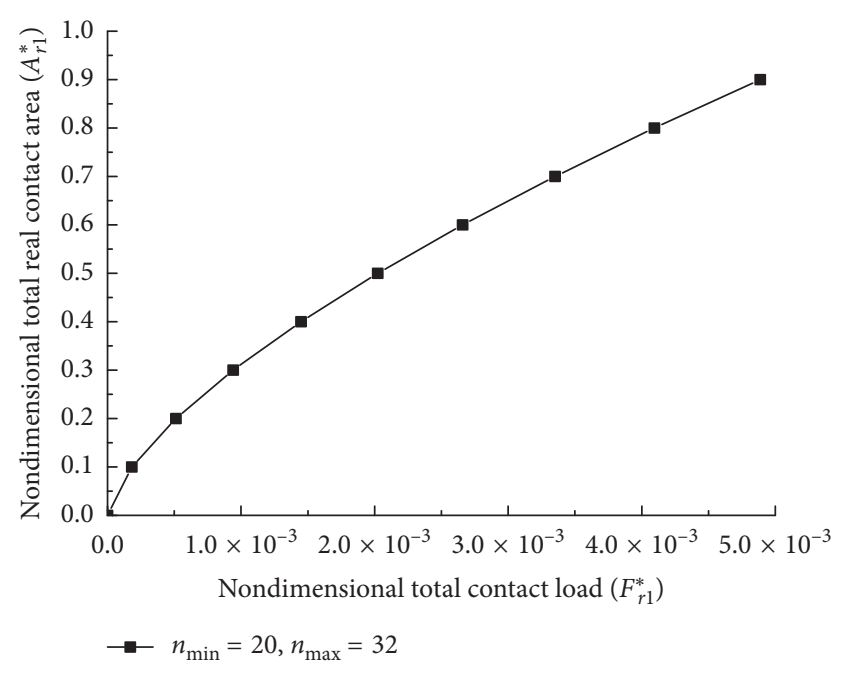

FIGURE 9: The relationship between dimensionless total real contact area and the dimensionless total contact loading for $20 \leq n \leq 32$.

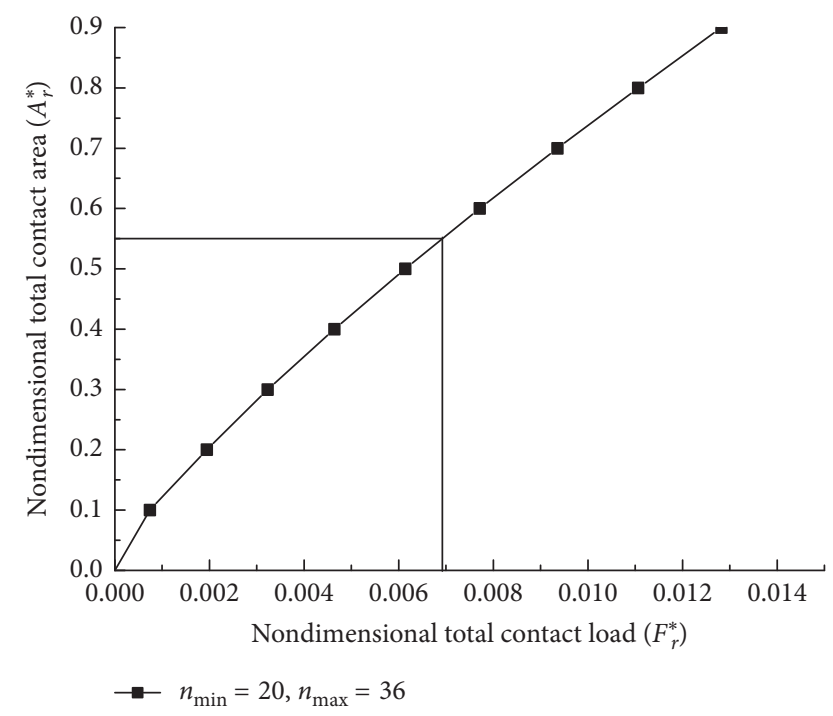

Figure 10: The relationship between dimensionless total real contact area and the dimensionless total contact loading for $20 \leq n \leq 36$.

the deformation characteristics of the asperity under different frequency exponents are obtained.

(2) The normal contact load of a single asperity in the elastoplastic stage is related to the hardness of the material. When the material yields, the hardness $H$ is not a constant, but a function related to the amount of deformation. In this paper, the limit mean geometric hardness is introduced to express the normal contact load of a single asperity in the first and second elastoplastic deformation stages considering the change of hardness.

(3) The relationship between the contact load and contact area of a single asperity in the first and second elastoplastic deformation stages considering

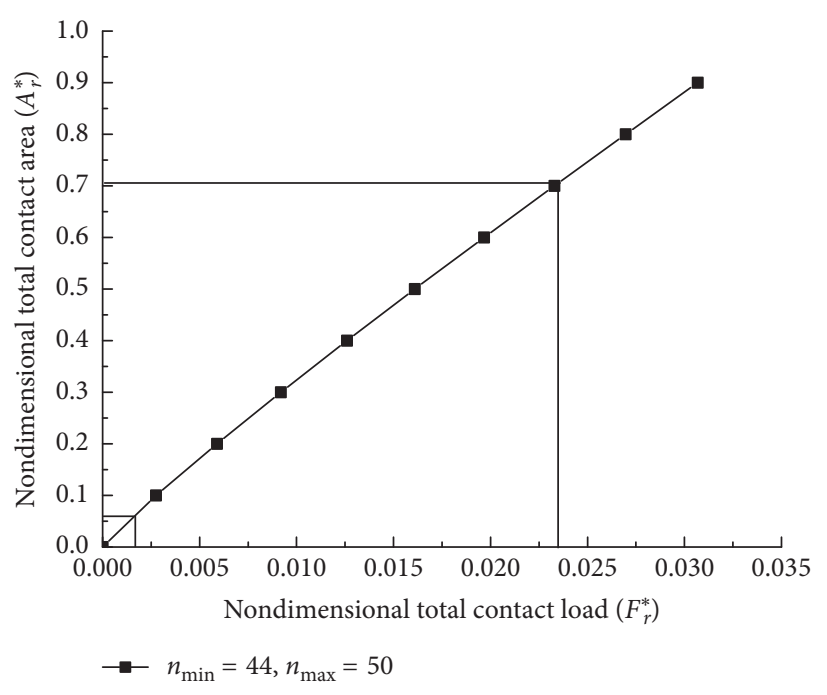

Figure 11: The relationship between dimensionless total real contact area and the dimensionless total contact loading for $44 \leq n \leq 50$.

the change of material hardness and not considering the change of hardness is compared, respectively. When the contact area of the asperity is the same, the contact load with the change of hardness is smaller than that without the change of hardness, and the difference between them increases with the increase of deformation.

(4) The limit mean geometric hardness of a single asperity is related to the contact area, fractal dimension, and frequency exponent during the elastoplastic deformation stage, and the limit mean geometric hardness increases with the increase of the contact area. When the frequency exponent is constant, the relationship between the limit mean geometric hardness and the contact area of the asperity is related to the fractal dimension. The larger the fractal dimension is, the more obvious the relationship curve between them is. When the fractal dimension is constant, the relationship between the limit mean geometric hardness of the asperity and the contact area is related to the frequency exponent. The smaller the frequency exponent is, the more obvious the change of the curve is.

(5) The relationship between dimensionless total real contact area and the dimensionless total contact loading of joint interface at each deformation stage is obtained by introducing the pushing coefficient, and the critical pushing coefficient exists. When the pushing coefficient exceeds this value, some asperities begin to deform in the next stage, and the inflection point appears on the relationship curve.

(6) The current model still has some limitations on the applicable scope of materials, and further research and improvement are needed to make it more applicable in the future. 


\section{Nomenclature}

$\omega_{\mathrm{n}}: \quad$ Interference of the asperity

$\omega_{\text {nec }}: \quad$ Elastic critical interference of the asperity

$\omega_{\text {nepc }}: \quad$ First elastoplastic critical interference of the asperity

$\omega_{\text {npc }}: \quad$ Second elastoplastic critical interference of the asperity

$a_{\mathrm{n}}: \quad$ Contact area of the asperity

$a_{\text {nec: }} \quad$ Elastic critical contact area of the asperity

$a_{\text {nepc }}: \quad$ First elastoplastic critical contact area of the asperity

$a_{\mathrm{npc}}: \quad$ Second elastoplastic critical contact area of the asperity

$f_{\text {ne }}$ Normal load in the elastic deformation of a single asperity

$f_{\text {nec }}$ : Normal contact load for $\omega=\omega_{\text {nec }}$

$f_{\text {nep } 1}$ : Normal contact load of a single asperity in the first elastoplastic stage

$f_{\text {nep2 }}$ : Normal contact load of a single asperity in the second elastoplastic stage

$f_{\text {np }}$ : Normal contact load of a single asperity in the full plastic deformation stage

$f_{\text {nep } 1}^{\prime}$ : Normal contact load of a single asperity in the first elastoplastic stage considering the change of hardness

$f_{\text {nep } 2}^{\prime}$ : Normal contact load of a single asperity in the second elastoplastic stage considering the change of hardness

$H_{\mathrm{G} 1}\left(a_{\mathrm{n}}\right)$ : Limit mean geometric hardness in the first elastoplastic deformation stage

$H_{\mathrm{G} 2}\left(a_{\mathrm{n}}\right)$ : Limit mean geometric hardness in the second elastoplastic deformation stage

$n_{\mathrm{ec}}: \quad$ Elastic critical frequency exponent

$n_{\text {epc }}: \quad$ First elastoplastic critical frequency exponent

$n_{\mathrm{pc}}$ : $\quad$ Second elastoplastic critical frequency exponent

$A_{\mathrm{r}}$ : $\quad$ Actual contact area of the joint interface

$A_{\mathrm{r} 1}$ : $\quad$ Actual contact area of the joint interface for $n_{\text {min }}<n \leq n_{\mathrm{ec}}$

$A_{\mathrm{r} 2}$ : $\quad$ Actual contact area of the joint interface for $n_{\mathrm{ec}}<n \leq n_{\mathrm{epc}}$

$A_{\mathrm{r} 3}$ : $\quad$ Actual contact area of the joint interface for $n_{\mathrm{epc}}<n \leq n_{\mathrm{pc}}$

$A_{\mathrm{r} 4}: \quad$ Actual contact area of the joint interface for $n_{\mathrm{pc}}<n$

$F_{\mathrm{r} 1}$ : Actual contact load of the joint interface for $n_{\min }<n \leq n_{\mathrm{ec}}$

$F_{\mathrm{r} 2}$ : $\quad$ Actual contact load of the joint interface for $n_{\mathrm{ec}}<n \leq n_{\mathrm{epc}}$

$F_{\mathrm{r} 3}$ : $\quad$ Actual contact load of the joint interface for $n_{\mathrm{epc}}<n \leq n_{\mathrm{pc}}$

$F_{\mathrm{r} 4}$ : Actual contact load of the joint interface for $n_{\mathrm{pc}}<n$

$p_{\text {ea }}(a)$ Average contact pressure of the asperity in elastic stage

$p_{\text {epal }}(a)$ : Average contact pressure of the asperity in the first elastoplastic deformation stage

$p_{\text {epa2 }}(a)$ : Average contact pressure of the asperity in the second elastoplastic stage.

\section{Data Availability}

The data used to support the findings of this study are available from the corresponding author upon request.

\section{Conflicts of Interest}

The authors declare that they have no conflicts of interest.

\section{Acknowledgments}

This work was supported by the National Natural Science Foundation of China (grant no. 51275328) and the Natural Science Foundation Project of Shanxi Province (grant no. 201601D011062).

\section{References}

[1] B. Zhao, S. Zhang, P. Wang, and Y. Hai, "Loading-unloading normal stiffness model for power-law hardening surfaces considering actual surface topography," Tribology International, vol. 90, pp. 332-342, 2015.

[2] J. Greenwood and J. Williamson, "Contact of nominally flat surfaces," Mathematical, Physical and Engineering Sciences, vol. 295, no. 1442, pp. 299-319, 1966.

[3] J. A. Greenwood and J. H. Tripp, "The contact of two nominally flat rough surfaces," Proceedings of the Institution of Mechanical Engineers, vol. 185, no. 1, pp. 625-633, 1970.

[4] W. R. Chang, I. Etsion, and D. B. Bogy, "An elastic-plastic model for the contact of rough surfaces," Journal of Tribology, vol. 109, no. 2, pp. 257-263, 1987.

[5] Y. Zhao, D. M. Maietta, and L. Chang, "An asperity microcontact model incorporating the transition from elastic deformation to fully plastic flow," Journal of Tribology, vol. 122, no. 1, pp. 86-93, 2000.

[6] L. Kogut and I. Etsion, "Elastic-plastic contact analysis of asphere and a rigid flat," Journal of Applied Mechanics, vol. 69, no. 5, pp. 657-662, 2002.

[7] I. Etsion, Y. Kligerman, and Y. Kadin, "Unloading of an elastic-plastic loaded spherical contact," International Journal of Solids and Structures, vol. 42, no. 13, pp. 3716-3729, 2005.

[8] Y. Kadin, Y. Kligerman, and I. Etsion, "Unloading an elasticplastic contact of rough surfaces," Journal of the Mechanics and Physics of Solids, vol. 54, no. 12, pp. 2652-2674, 2006.

[9] Y. Kadin, Y. Kligerman, and I. Etsion, "Multiple loadingunloading of an elastic-plastic spherical contact," International Journal of Solids and Structures, vol. 43, no. 22-23, pp. 7119-7127, 2006.

[10] A. Majumdar and B. Bhushan, "Role of fractal geometry in roughness characterization and contact mechanics of surfaces," Journal of Tribology, vol. 112, no. 2, pp. 205-216, 1990.

[11] A. Majumdar and C. L. Tien, "Fractal characterization and simulation of rough surfaces," Wear, vol. 136, no. 2, pp. 313-327, 1990.

[12] A. Majumdar and B. Bhushan, "Fractal model of elastic-plastic contact between rough surfaces," Journal of Tribology, vol. 113, no. 1, pp. 1-11, 1991.

[13] S. Wang and K. Komvopoulos, "A fractal theory of the interfacial temperature distribution in the slow sliding regime: Part I-elastic contact and heat transfer analysis," Journal of Tribology, vol. 116, no. 4, pp. 812-822, 1994.

[14] S. Wang and K. Komvopoulos, "A fractal theory of the interfacial temperature distribution in the slow sliding regime: 
Part II-multiple domains, elastoplastic contacts and applications," Journal of Tribology, vol. 116, no. 4, pp. 824-832, 1994.

[15] Y. Morag and I. Etsion, "Resolving the contradiction of asperities plastic to elastic mode transition in current contact models of fractal rough surfaces," Wear, vol. 262, no. 5-6, pp. 624-629, 2007.

[16] H. Tian, X. Zhong, and C. Zhao, "One loading model of joint interface considering elastoplastic and variation of hardness with surface depth," Journal of Mechanical Engineering, vol. 51, no. 5, pp. 90-104, 2015.

[17] Y. Yuan, Y. Cheng, K. Liu et al., "A revised Majumdar and Bushan model of elastoplastic contact between rough surfaces," Applied Surface Science, vol. 425, pp. 1138-1157, 2017.

[18] R. S. Sayles and T. R. Thomas, "Surface topography as a nonstationary random process," Nature, vol. 271, no. 5644, pp. 431-434, 1978. 


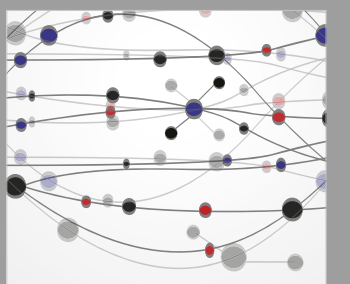

The Scientific World Journal
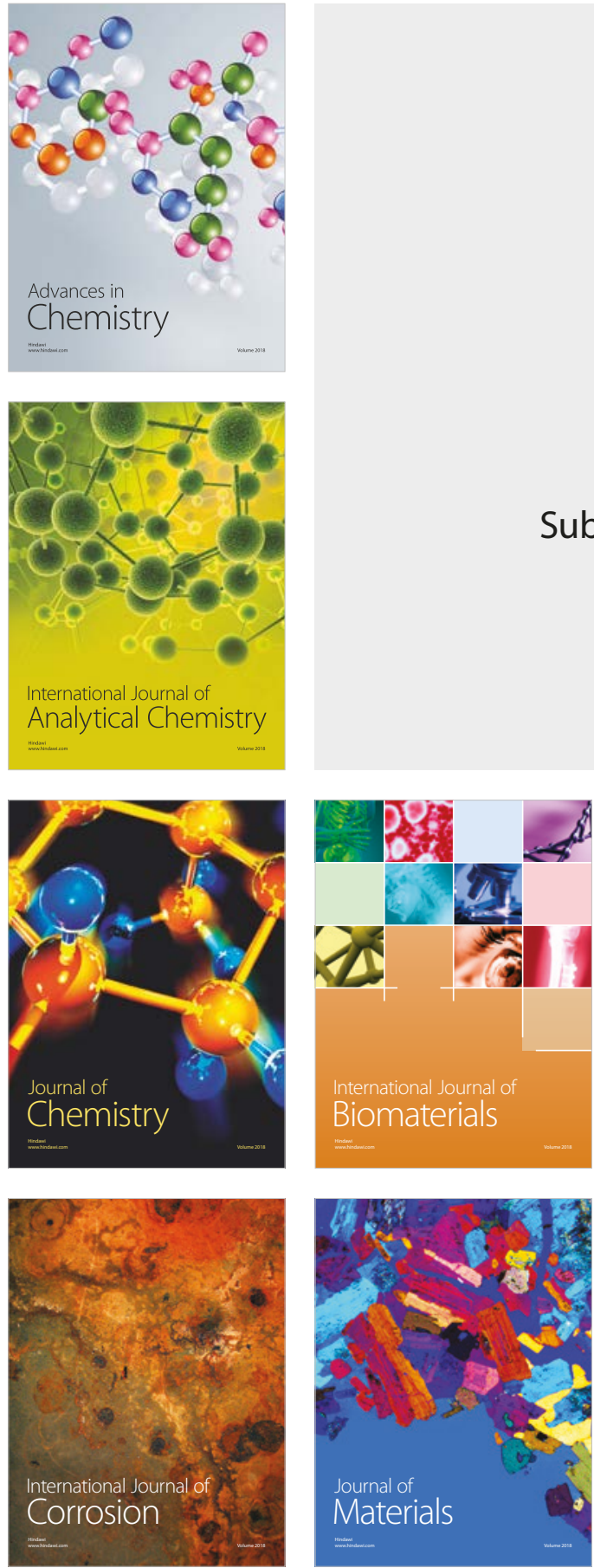

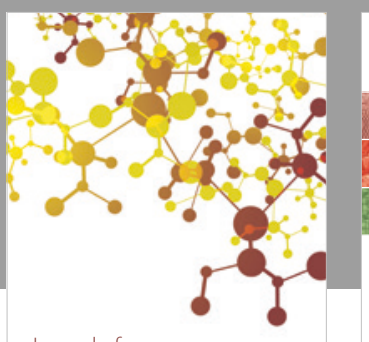

Journal of

Applied Chemistry
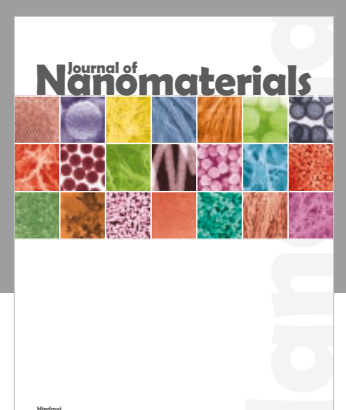

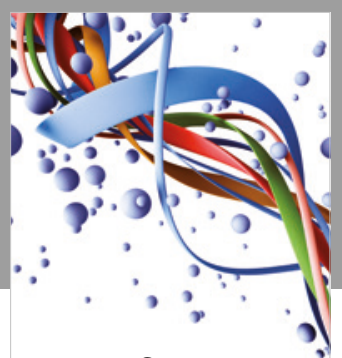

Scientifica

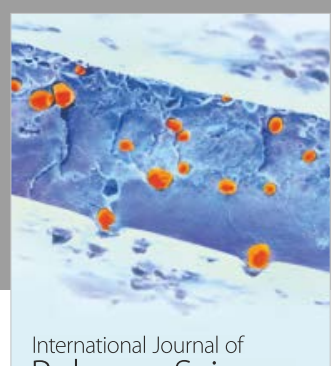

Polymer Science

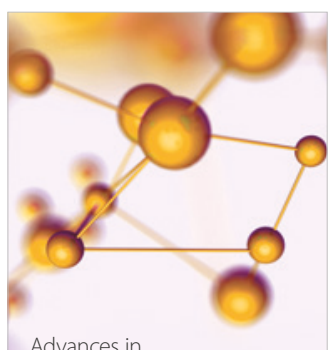

Physical Chemistry
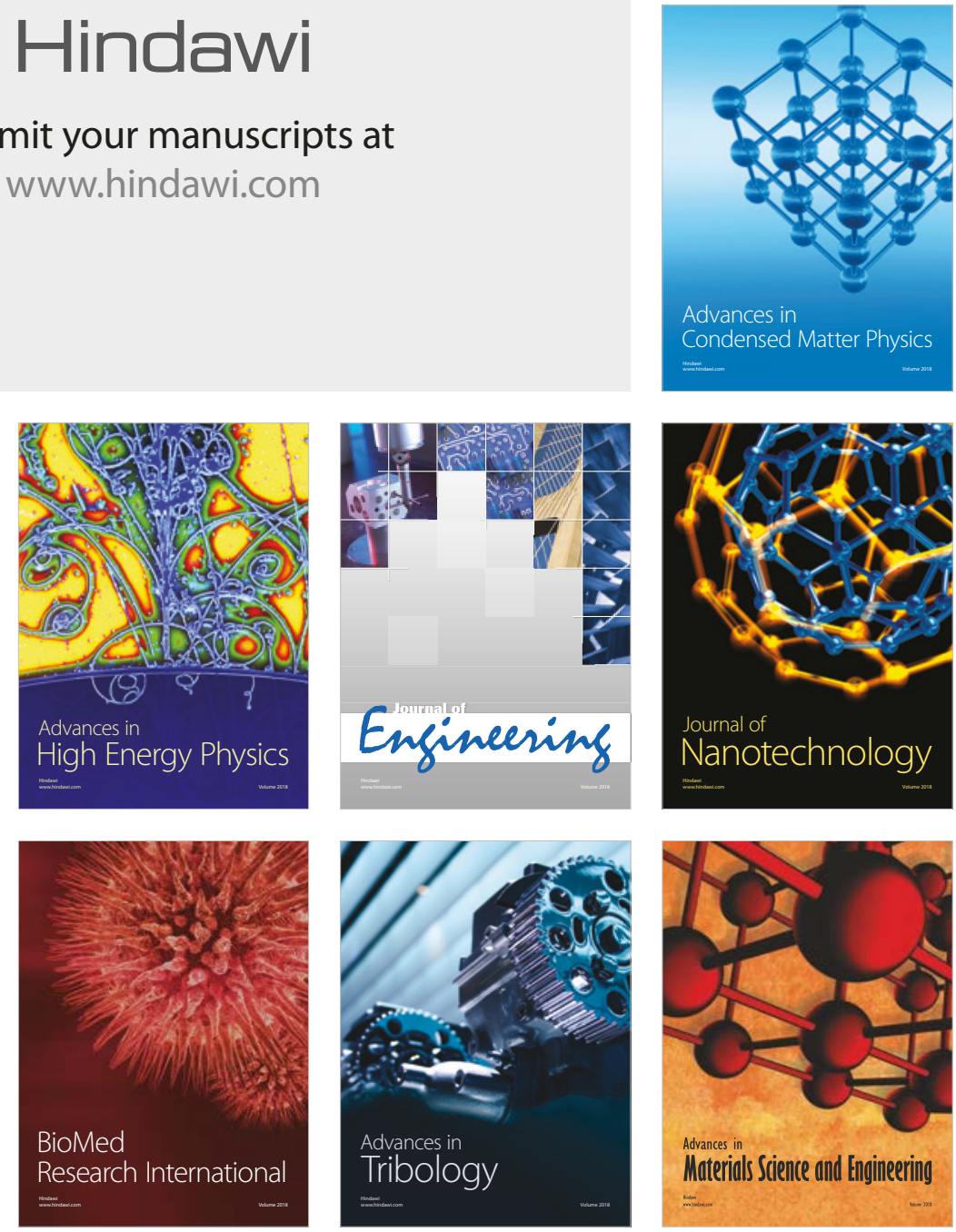